\title{
Parâmetros fisiológicos de uma sessão de exercício calistênico intermitente de alta intensidade em praticantes avançados
}

Physiological parameters of a high intensity intermittent calistenic exercise in advanced practitioners

Parámetros fisiológicos de un ejercicio calisténico intermitente de alta intensidad en practicantes avanzados

Tharciano Luiz Teixeira Braga da Silva ORCID: https://orcid.org/0000-0001-5467-7875 Centro Universitário Estácio de Sergipe, Brasil E-mail: tharcianoluiz@gmail.com Jadisson Gois da Silva ORCID: https://orcid.org/0000-0003-0089-4852 Universidade Federal de Santa Catarina, Brasil E-mail: jadissoned.fisica2014@ outlook.com André Luiz Silva Santos ORCID: https://orcid.org/0000-0001-7175-7033 Universidade Federal de Sergipe, Brasil E-mail: andrelss.edf@gmail.com

Mycaelle Emily Santana da Cruz ORCID: https://orcid.org/0000-0002-5909-457X Centro Universitário Estácio de Sergipe, Brasil E-mail: emilly1095@hotmail.com

Fabricio Nunes Macedo

ORCID: https://orcid.org/0000-0002-6810-7766 Centro Universitário Estácio de Sergipe, Brasil E-mail: briciumacedo@gmail.com

Vitor Ulisses de Melo

ORCID: https://orcid.org/0000-0002-0614-5900 Universidade Federal de Sergipe, Brasil E-mail: vumelo@gmail.com

Michael Nadson Santos Santana ORCID: https://orcid.org/0000-0003-2121-1192 Centro Universitário Estácio de Sergipe, Brasil E-mail: micsantos@gmail.com

Marcelo Mendonça Mota

ORCID: https://orcid.org/0000-0001-8452-8061 Centro Universitário Estácio de Sergipe, Brasil E-mail: mota.marcelo@gmail.com

\begin{abstract}
Resumo
O exercício calistênico intermitente de alta intensidade (ECIAI) é considerado um método eficaz e de baixo custo para a melhora da aptidão física e da composição corporal. O objetivo do estudo foi avaliar os efeitos agudos de uma sessão de ECIAI sobre percepção subjetiva ao esforço (PSE), saturação de oxigênio $\left(\mathrm{SpO}_{2}\right)$ e variáveis hemodinâmicas em praticantes avançados. Foram avaliados 8 homens praticantes da modalidade Cross Training e CrossFit ${ }^{\circledR}$. O protocolo consistiu em 4 séries com o máximo de execuções durante 30 segundos, seguido de 2 minutos de descanso. A PSE foi avaliada imediatamente após cada série. A $\mathrm{SpO}_{2}$, frequência cardíaca (FC), pressão arterial sistólica (PAS) e pressão arterial diastólica (PAD) foram aferidas no repouso e imediatamente após cada série. Os valores foram expressos como a média \pm desvio padrão da média. Não ocorreu diferenças entre as séries na PSE. Houve aumento da FC (bpm) após a realização das séries 1, 2, 3 e 4. Quanto a PAS (mmHg), foi observado aumento após as séries 2 e 3 quando comparado ao repouso. A PAD (mmHg) não foi alterada. A PAM (mmHg) aumenta imediatamente após série 2 e 3 quando comparado ao repouso. O Duplo Produto também apresentou aumento imediatamente após todas as séries quando comparado ao repouso. Os resultados do presente estudo indicam que o ECIAI promoveu importantes ajustes cardiorrespiratórios em adultos com experiência nessa modalidade de exercício. Estes efeitos caracterizam um esforço físico vigoroso e reforçam a importância do controle fisiológico da carga interna durante a sessão de treinamento.
\end{abstract}

Palavras-chave: Treinamento intervalado de alta intensidade; Esforço físico; Sistema cardiovascular. 


\begin{abstract}
High intensity intermittent calisthenic exercise (ECIAI) is considered an effective and low cost method for improving physical fitness and body composition. The aim of the study was to evaluate the acute effects of an ECIAI session on subjective perception of effort (RPE), oxygen saturation (SpO2) and hemodynamic variables in advanced practitioners. 8 men practicing Cross Training and CrossFit ${ }^{\circledR}$ were evaluated. The protocol consisted of 4 sets with maximum executions for 30 seconds, followed by 2 minutes of rest. PSE was assessed immediately after each series. $\mathrm{SpO}$, heart rate (HR), systolic blood pressure (SBP) and diastolic blood pressure (DBP) were measured at rest and immediately after each series. Values were expressed as the mean \pm standard deviation from the mean. There were no differences between series in PSE. There was an increase in HR (bpm) after series 1, 2, 3 and 4. As for SBP (mmHg), an increase was observed after series 2 and 3 when compared to rest. The DBP $(\mathrm{mmHg})$ was not changed. MAP $(\mathrm{mmHg})$ increases immediately after series 2 and 3 when compared to rest. The Double Product also increased immediately after all series when compared to rest. The results of the present study indicate that ECIAI promoted important cardiorespiratory adjustments in adults with experience in this type of exercise. These effects characterize a vigorous physical effort and reinforce the importance of the physiological control of the internal load during the training session.
\end{abstract}

Keywords: High-intensity interval training; Physical exertion; Cardiovascular system.

\title{
Resumen
}

El ejercicio calisténico intermitente de alta intensidad (ECIAI) se considera un método eficaz y de bajo costo para mejorar la condición física y la composición corporal. El objetivo del estudio fue evaluar los efectos agudos de una sesión de ECIAI sobre la percepción subjetiva del esfuerzo (PSE), la saturación de oxígeno (SpO2) y las variables hemodinámicas en practicantes avanzados. Se evaluaron 8 hombres practicando la modalidad Cross Training y CrossFit@. El protocolo consistió en 4 series con ejecuciones máximas durante 30 segundos, seguidas de 2 minutos de descanso. La PSE se evaluó inmediatamente después de cada serie. Se midieron SpO2, frecuencia cardíaca (FC), presión arterial sistólica (PAS) y presión arterial diastólica (PAD) en reposo e inmediatamente después de cada serie. Los valores se expresaron como el promedio \pm desviación estándar del promedio. No hubo diferencias entre las series en el PSE. Hubo un aumento de la FC (lat/min) después de realizar las series 1, 2, 3 y 4. En cuanto a la PAS (mmHg), se observó un aumento después de las series 2 y 3 con respecto al reposo. La PAD (mmHg) no se modificó. La PAM $(\mathrm{mmHg})$ aumenta inmediatamente después de las series 2 y 3 en comparación con el reposo. El Producto Doble también mostró un aumento inmediatamente después de toda la serie en comparación con el descanso. Los resultados del presente estudio indican que el ECIAI promovió importantes ajustes cardiorrespiratorios en adultos con experiencia en este tipo de ejercicio. Estos efectos caracterizan un esfuerzo físico vigoroso y refuerzan la importancia del control fisiológico de la carga interna durante la sesión de entrenamiento.

Palabras clave: Entrenamiento en intervalos de alta intensidad; Esfuerzo físico; Sistema cardiovascular.

\section{Introdução}

O exercício calistênico intermitente de alta intensidade (ECIAI) vem ganhando popularidade por ser considerado um método eficaz e de baixo custo para a melhora da aptidão física e da composição corporal (Gist et al., 2014; Machado et al., 2017; Schaun \& Vecchio, 2018; Schaun et al., 2018). Além disso, o ECIAI é também descrito como um exercício que promove estímulos vigorosos no corpo inteiro em curto espaço de tempo (Gist et al., 2014; Paula et al., 2020; Tucker et al., 2015; Weston et al., 2014).

McRae et al. (2012) relataram que um programa de ECIAI é mais benéfico no aumento da aptidão cardiovascular do que um programa de exercício aeróbico contínuo de intensidade moderada. De forma similar, Gist et al. (2014) demonstraram que uma sessão de ECIAI de baixo volume e alta intensidade pode promover adaptações cardiorrespiratórias e metabólicas equivalentes às relatadas em estudos usando o sprint interval cycling (SIC). Durante a realização de exercício de alta intensidade, pode ser visto um aumento exponencial nos valores da frequência cardíaca (FC) e pressão arterial (PA) com a finalidade de demandar um aporte sanguíneo adequado às musculaturas recrutadas no exercício (Forjaz et al., 1998; Forjaz \& Tinucci, 2000).

Também é observado que o exercício intermitente de alta intensidade pode reduzir a saturação de oxigênio $\left(\mathrm{SpO}_{2}\right)$ (Nummela et al., 2002; Peeling \& Andersson, 2011). A literatura indica que a percepção subjetiva ao esforço (PSE) durante uma sessão ECIAI com exercícios do tipo burpees podem ser consideradas de grande esforço físico (Gist et al., 2014) Diante disso, para minimizar os riscos de eventos cardiovasculares durante o exercício, se faz necessária à utilização de recursos que 
possam auxiliar no controle da carga de trabalho do coração (Kalra \& Roitman, 2007). Assim, é possível quantificar a carga do exercício por meio da $\mathrm{PSE}, \mathrm{SpO}_{2}$ e $\mathrm{FC}$, sendo estas, variáveis fisiológicas que permitem compreender o estado físico do praticante durante o exercício.

Até onde sabemos, nenhuma pesquisa foi conduzida para determinar o comportamento da $\mathrm{SpO}_{2}$ durante um protocolo de ECIAI. O conhecimento do comportamento dessa resposta aguda ajudará a melhorar a aplicabilidade prática deste tipo de exercício e favorecer um maior controle sobre as adaptações fisiológicas, como também, os seus riscos e benefícios para a saúde. Nós hipotetizamos que o ECIAI promove respostas cardiorrespiratórias suficientes para ser classificado como um exercício físico vigoroso e que precisa ser monitorado durante a sua prática diária. Diante disso, o objetivo do presente estudo foi avaliar os efeitos agudos de uma sessão de ECIAI sobre percepção subjetiva ao esforço (PSE), saturação de oxigênio $\left(\mathrm{SpO}_{2}\right)$ e variáveis hemodinâmicas em praticantes avançados.

\section{Métodos}

\section{Tipo de estudo}

O presente estudo é de caráter quantitativo, transversal e do tipo experimental (Thomas et al., 2012).

\section{Participantes}

Foram avaliados 8 homens praticantes do ECIAI por meio da modalidade Cross Training e CrossFit ${ }^{\circledR}$. Todos possuíam mais de um ano de experiência nestas modalidades, treinavam regularmente com grande esforço físico e com frequência mínima de 3 vezes por semana. Todos os sujeitos foram familiarizados com os procedimentos empregados. Não participaram do presente estudo aqueles que utilizassem fármacos e/ou recursos nutricionais ergogênicos, assim como praticantes que apresentaram lesões ortopédicas ou distúrbios metabólicos nos últimos 6 meses. O presente estudo foi aprovado pelo Comitê de Ética em Pesquisa (CAEE: 83636918.6.0000.8079) e está de acordo com a resolução 510/2016 do Conselho Nacional de Saúde.

\section{Procedimentos}

Todos os procedimentos experimentais foram realizados em apenas uma visita ao laboratório. As instruções do préexperimento direcionaram os indivíduos a chegarem hidratados e o não consumo de bebidas com cafeína, álcool, tabaco, estimulantes e exercícios físicos por pelo menos 48 horas antes da chegada ao laboratório. Os ensaios experimentais ocorreram no mesmo período do dia (08:00 às 11:00 horas) em um ambiente com temperatura estável ( $\left.\cong 22^{\circ} \mathrm{C}\right)$. No dia do experimento os participantes assinaram o Termo de Consentimento Livre e Esclarecido (TCLE) e foram explicadas pela equipe de pesquisa as instruções sobre os procedimentos experimentais. Não ocorreu a familiarização com o protocolo de ECIAI por já ser um exercício da rotina de treinamentos dos participantes.

Antes do início do protocolo de exercício foram registrados os dados antropométricos para a caracterização da amostra. Além disso, foram avaliados os parâmetros de $\mathrm{SpO}_{2}$ e da FC dos indivíduos depois de 20 minutos de repouso (Figura 1). Após as medidas de base, foi feito um aquecimento e logo em seguida foi realizado o protocolo de ECIAI. As medidas de $\mathrm{SpO}_{2}$ e da $\mathrm{FC}$ foram novamente coletadas imediatamente após cada série de exercício com o indivíduo sentado em uma cadeira. Os sujeitos usaram um monitor de FC durante todo o protocolo para permitir o monitoramento contínuo e foi usada a escala de PSE de Borg (1-10) modificada por Foster (1998) para medir o esforço físico do exercício. Os participantes foram estimulados verbalmente para atingir o máximo de movimentos possíveis durante cada série e instruídos a fornecer informações contínuas sobre o esforço físico, fadiga, falta de ar incapacitante, dor articular e angina. 
Figura 1: Protocolo experimental.

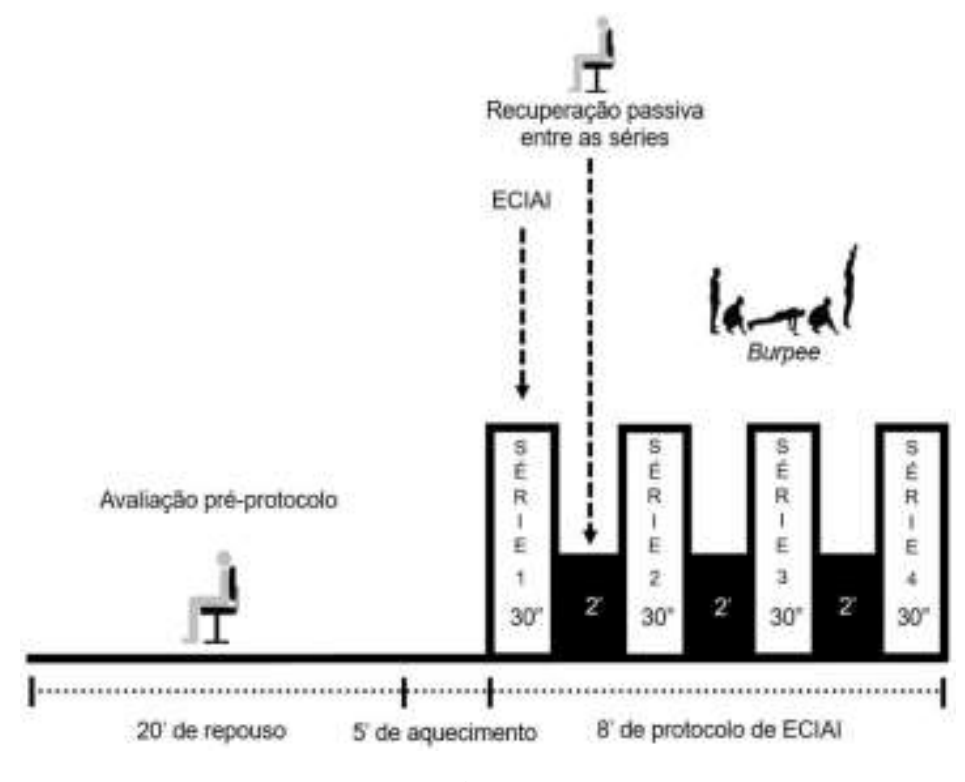

Fonte: Autores.

\section{Avaliação antropométrica}

Para a caracterização da amostra a massa corporal foi aferida por meio de uma balança portátil digital (BC 558 Tanita®, Japão), com precisão de $0,1 \mathrm{~kg}$ e capacidade total de $150 \mathrm{~kg}$. A estatura também foi avaliada para calcular o índice de massa corporal (IMC) utilizando o antropômetro portátil (Body Meter 208, Seca ${ }^{\circledR}$, United Kingdom), com precisão de $1 \mathrm{~cm}$. O IMC foi calculado a partir da massa corporal, em quilos, dividida pelo quadrado da estatura em metros. Foi realizada a aferição das dobras cutâneas por meio de um adipômetro digital científico (Prime Med DG+®), em uma sala climatizada a $\cong 22^{\circ} \mathrm{C}$. O protocolo utilizado para determinar a massa gorda, a massa magra e o percentual de gordura (\% G) foi o de Pollock de 7 dobras para homens e todas as medidas foram feitas em triplicatas. As variáveis antropométricas e de composição corporal foram avaliadas antes do protocolo experimental.

\section{Avaliação da percepção subjetiva ao esforço, quantidade de burpees, saturação de oxigênio e frequência cardíaca}

$\mathrm{Na}$ avaliação da PSE foi usada à escala de Borg modificada de 10 pontos ao final de cada série de ECIAI (Foster, 1998; Scott et al., 2015). A quantidade de burpees foi medida e foram considerados na contagem os movimentos completos até o final do tempo estipulado de cada série de ECIAI. A $\mathrm{SpO}_{2}$ foi avaliada no dedo indicador esquerdo por meio de um oxímetro de dedo (More fitness $\left.{ }^{\circledR}\right)$ (Neto et al., 2016). A FC foi monitorada continuamente por um monitor Polar® V800 decodificado. $\mathrm{A} \mathrm{SpO}_{2}$ e a FC foram avaliadas após 20 minutos de repouso e imediatamente após cada série do protocolo de ECIAI com o indivíduo em recuperação passiva sentado em uma cadeira.

\section{Exercício calistênico intermitente de alta intensidade}

Antes do protocolo de exercício foi realizado um aquecimento composto por 10 agachamentos, 10 apoios no solo e 5 Burpees. O protocolo de ECIAI foi caracterizado pela realização 4 séries de 30 segundos de burpees (máximo de repetições possíveis), intercalados por 2 minutos de recuperação passiva com o indivíduo sentado em uma cadeira. Durante a recuperação passiva foram coletados a PSE, $\mathrm{SpO}_{2}$ e a FC. O exercício do Burpee é iniciado na posição ortostática com os braços ao lado do corpo (Figura 2). Em seguida, flexionam-se os joelhos (agachamento) e apoiam-se as mãos no solo em frente aos pés. Rapidamente, estendem-se o quadril e os joelhos empurrando os pés para trás para então flexionar e estender os cotovelos 
(apoio no solo), retornando os pés para a posição de agachamento. Por fim, estendem-se os joelhos e quadris saltando o mais alto possível com os braços estendidos (Gist et al., 2014).

Figura 2: Fases de um movimento de Burpee.

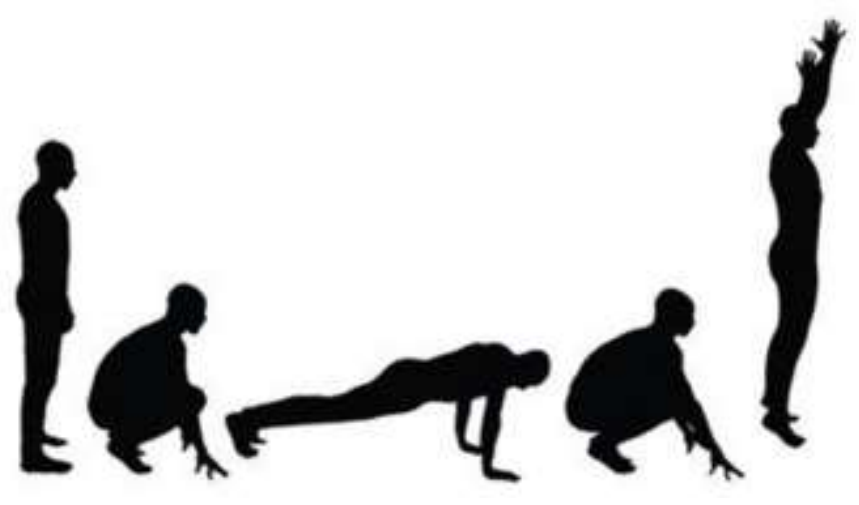

Fonte: https://horadotreino.com.br/exercicio-burpee/

\section{Análise estatística}

Os valores foram expressos como a média \pm desvio padrão da média. A normalidade foi verificada pelo teste de Shapiro-Wilk. Os testes de análise de variância (ANOVA de "uma via") seguido do pós-teste de Bonferroni foram utilizados para avaliar a significância das diferenças entre as médias. Os valores foram considerados estatisticamente significativos quando p< 0,05. Em todos estes procedimentos foi utilizado o programa estatístico GraphPad Prism versão 6.0.

\section{Resultados}

\section{Caracterização da amostra}

As características antropométricas da amostra podem ser observadas na Tabela 1. São descritas as variáveis relacionadas à idade, estatura, massa corporal, índice de massa corporal (IMC), percentual de gordura, massa gorda e massa magra dos praticantes de ECIAI que participaram do presente estudo.

Tabela 1: Caracterização dos praticantes de ECIAI no início do protocolo.

\begin{tabular}{lc}
\hline \multicolumn{1}{c}{ VARIÁVEIS } & RESULTADOS \\
\hline Idade (anos) & $23,75 \pm 3,7$ \\
Estatura (cm) & $174,25 \pm 5,0$ \\
Massa corporal (kg) & $71,79 \pm 6,8$ \\
IMC (kg/m ${ }^{2}$ ) & $23,88 \pm 2,4$ \\
Percentual de gordura (\%) & $7,65 \pm 0,5$ \\
Massa Gorda (kg) & $6,10 \pm 1,8$ \\
Massa Magra $(\mathbf{k g})$ & $66,47 \pm 5,2$ \\
\hline
\end{tabular}

Nota: Os resultados são expressos como a média \pm o erro padrão da média. Abreviações - cm: centímetros; kg: quilograma; IMC: índice de massa corporal; $\mathrm{kg} / \mathrm{m}^{2}$ : quilograma por metro quadrado; \%: percentual. Fonte: Autores. 


\section{Efeito do ECIAI sobre a PSE, quantidade de burpees, $\mathrm{SpO}_{2}$ e FC}

Como demonstrado na Figura 3, não foram observadas diferenças entre as séries na avaliação da PSE (Figura 3A) e na quantidade de burpees (Figura 3B) executadas pelos praticantes de ECIAI.

Figura 3: Monitoramento da Percepção Subjetiva ao Esforço (PSE, figura 3A) e quantidade de Burpee (Repetições, figura 3B) dos praticantes de ECIAI imediatamente após cada série.
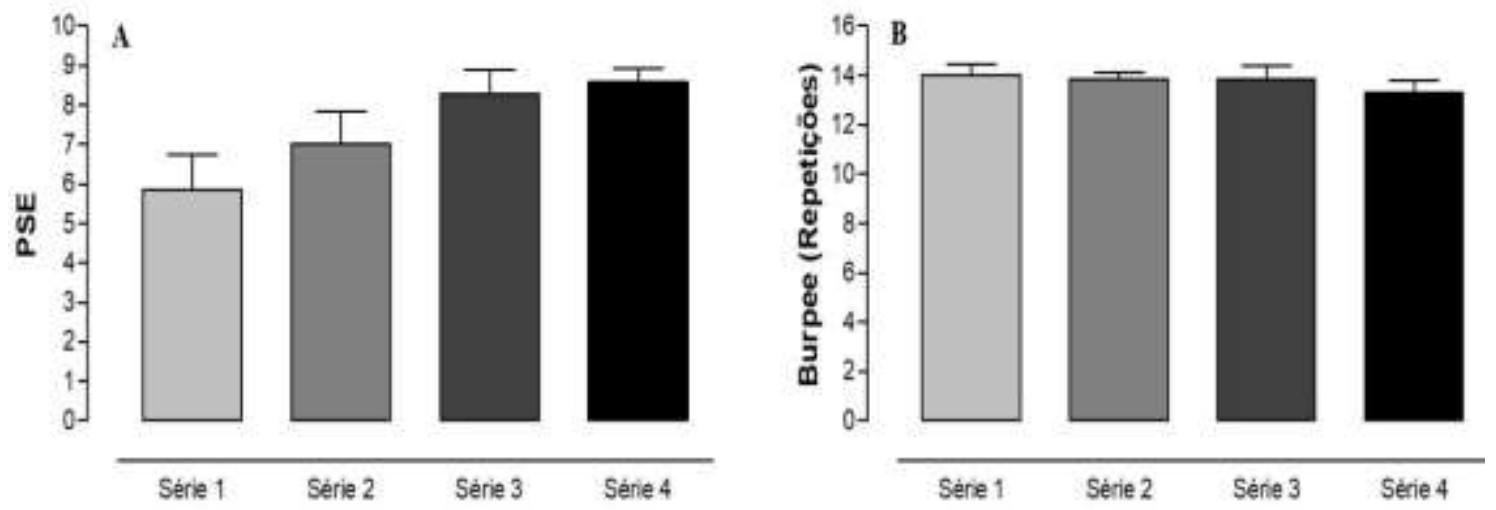

Nota: Os resultados são expressos como a média \pm desvio padrão da média. Para análise estatística foi utilizada ANOVA de uma via seguido do pós-teste de Bonferroni. Fonte: Autores.

Já na Figura 4, a $\mathrm{SpO}_{2}$ dos praticantes de ECIAI apresentaram um decréscimo significativo $(\mathrm{p}<0,05)$ imediatamente após a quarta série de Burpees $(97 \pm 1,2 \%)$ quando comparada com o repouso $(99 \pm 0,3 \%)$.

Figura 4: Monitoramento da Saturação de Oxigênio $\left(\mathrm{SpO}_{2}\right)$ dos praticantes de ECIAI no repouso e imediatamente após cada série de burpees.

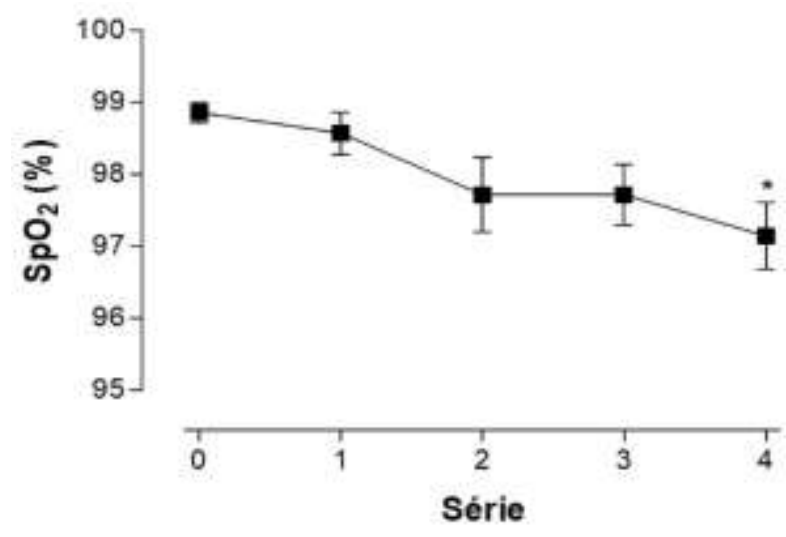

Nota: Os resultados são expressos como a média \pm desvio padrão da média. Para análise estatística foi utilizada ANOVA de uma via seguido do pós-teste de Bonferroni. * p $<0,05$ vs repouso. Fonte: Autores.

Como demonstrado na Tabela 2, a FC dos praticantes de ECIAI obteve aumento significativo $(\mathrm{p}<0,001)$ imediatamente após a série 1 de Burpees (157,4 $\pm 11,8$ bpm) e nas subsequentes (série 2: 163,5 \pm 7,7 bpm; série 3: 167,4 \pm 7,9 bpm; série 4: 169,1 $\pm 8,7$ bpm) quando comparada com o repouso (60,8 $\pm 8,4$ bpm). 
Tabela 2: Frequência Cardíaca entre as sérias $(\mathrm{n}=8)$.

\begin{tabular}{lc}
\hline Avaliações & FC (bpm) \\
\hline Repouso & $60,8 \pm 8,49$ \\
Série 1 & $157,4 \pm 11,8^{* * *}$ \\
Série 2 & $163,5 \pm 7,7^{* * *}$ \\
Série 3 & $167,4 \pm 7,9^{* * *}$ \\
Série 4 & $169,1 \pm 8,7^{* * *}$ \\
\hline
\end{tabular}

Nota: Os valores são expressos como a média \pm desvio padrão da média. As diferenças estatísticas entre as médias intragrupo foram determinadas pela ANOVA de uma via seguido do pós-teste de Bonferroni. $* \mathrm{p}<0,05$ vs Repouso; ** $\mathrm{p}<0,01$ vs Repouso; ***p $<0,001$ vs Repouso. Abreviações: ECIAI, Exercício calistênico intermitente de alta intensidade; FC, Frequência cardíaca; bpm, batimentos por minuto. Fonte: Autores.

\section{Discussão}

Os resultados evidenciados neste estudo indicam que o protocolo utilizado foi capaz de promover importantes ajustes cardiorrespiratórios durante uma sessão de ECIAI em praticantes com experiência avançada nesta modalidade. A FC sofre um abrupto aumento durante todo o protocolo de exercício caracterizando desta forma um estímulo fisiológico vigoroso. São descritos diversos tipos de protocolos de exercícios intermitentes de alta intensidade (HIIT) que promovem diferentes respostas no sistema cardiovascular. Estes protocolos se diferenciam pela execução do movimento corporal, oscilações no tempo de estímulo e de recuperação (Gist et al., 2014; Schaun et al., 2018; Tucker et al., 2015).

Gist et al. (2014) compararam as respostas fisiológicas de dois protocolos de HIIT: o sprint interval cycling (SIC) e o ECIAI. Foi observado que o ECIAI provoca modificações cardiorrespiratórias vigorosas e ajustes fisiológicos semelhantes às relatadas no protocolo de SIC. O protocolo SIC consistiu em pedalar o mais rápido possível durante 30 segundos com resistência, seguido de 4 minutos de intervalo pedalando sem resistência. Já o protocolo de ECIAI, consistiu em realizar o máximo de Burpees possíveis por 30 segundos, seguido de 4 minutos de recuperação ativa (marchar no mesmo lugar em ritmo auto selecionado). Vale destacar que o protocolo de ECIAI adotado no presente estudo possui diferenças entre os já publicados na literatura com relação a um menor intervalo entre as séries ( 2 minutos) e a utilização de pausa passiva (sentado em uma cadeira) para a recuperação entre as séries.

Os resultados demonstraram que não ocorreram diferenças na PSE ou na quantidade de burpees durante o protocolo de ECIAI entre as séries. Embora a PSE não tenha apresentado diferenças, pode ser observado que na última série do ECIAI chegou próximo a valores de 8, que é classificado na escala de "Borg modificada" (1-10) como muito intenso. Gist et al. (2014) também observaram resultados semelhantes sobre a PSE. Eles observaram que quando é realizado o máximo de Burpees possíveis por 30 segundos a PSE medida pela escala de "Borg clássica" (6-20) chega a aproximadamente 14-17 (difícil-muito difícil).

Dessa forma, corroborando com os dados da literatura parece que o protocolo de ECIAI adotado no presente estudo produz uma PSE vigorosa. Também podemos perceber com os resultados que a quantidade de Burpees executados em cada série foram de aproximadamente 13-14 em 30 segundos de exercício. Vale destacar que todos os participantes foram estimulados verbalmente para tentar atingir o máximo de Burpees possíveis em todas as séries do protocolo de ECIAI e com isso, conseguiram manter a mesma performance ao longo das quatro séries de Burpees. Além da PSE, a SpO 2 e a FC, são variáveis eficientes para o controle da carga interna de treinamento de um exercício físico vigoroso.

A $\mathrm{SpO}_{2}$ pode ser descrita como a quantidade de oxigênio expressa em percentuais que é transportado no sangue para os tecidos. A variação da queda da $\mathrm{SpO}_{2}$ dependente de diversos fatores inclusive a modalidade de exercício executada (Galy et al., 2005). É descrito que o exercício intermitente de alta intensidade promove redução na $\mathrm{SpO}_{2}$ e como consequência, uma 
menor disponibilidade de oxigênio para o músculo esquelético durante o exercício (Nummel et al, 2002; Peeling \& Andersson, 2011).

Face ao contexto, nossos resultados também demonstraram queda na $\mathrm{SpO}_{2}$. Efeitos semelhantes na $\mathrm{SpO}_{2}$ podem ser observados em estudos com indivíduos saudáveis que foram submetidos a um protocolo de exercício resistido ou indivíduos submetidos a um protocolo de combate simulado de jiu-jitsu (Scot et al, 2015; Santos et al., 2018). Dentro do nosso conhecimento este é o primeiro estudo a avaliar efeitos do ECIAI sobre a $\mathrm{SpO}_{2}$ de forma não invasiva. Dessa forma, podemos sugerir que o protocolo de ECIAI promoveu um grande estresse cardiorrespiratório após a quarta série de Burpees que foi possivelmente provocado pela queda da $\mathrm{SpO}_{2}$.

Também verificamos que o ECIAI foi capaz de promover um abrupto aumento da FC em todas as séries de Burpees. Vale salientar que o protocolo de ECIAI adotado no presente estudo foi composto de intervalos de 2 minutos entre as séries seguidos de pausas passivas com o indivíduo sentado em uma cadeira. Parece que esse tipo de recuperação entre as séries de Burpees não foi capaz de amortizar as respostas da FC imediatamente após o exercício. Em um protocolo similar ao nosso, Gist et al. (2014) utilizaram 4 minutos de recuperação ativa entre as séries de Burpees com os indivíduos em marcha estacionária e obteve resultados que corroboram com os nossos. Eles classificaram o protocolo de ECIAI avaliado como um exercício de intensidade vigorosa (64-90\% da captação máxima de oxigênio; 77-95\% da FC máxima).

Em uma perspectiva de aplicabilidade prática os nossos resultados demonstram que o exercício do tipo HIIT executado por meio de Burpees em uma sessão, com baixo volume, alta intensidade e pausas passivas é de característica fisicamente vigorosa mesmo quando realizado por praticantes com experiência. Já é relatado na literatura que o ECIAI promove ajustes cardiorrespiratórios e metabólicos vigorosos equivalentes aos encontrados em protocolos de SIC (Gist et al., 2014). Os exercícios com o peso corporal (exercícios calistênicos) vêm ocupando lugar de destaque no cenário mundial por ser um exercício de baixo custo e que promove rápidas melhoras no condicionamento físico com o mínimo de tempo gasto para o treinamento diário (Chaves et al., 2020; Gist et al., 2014).

Apesar disso, devemos levar em consideração que o protocolo de ECIAI realizado da maneira descrita neste estudo promove estímulo cardiorrespiratório vigoroso mesmo quando são realizados 2 minutos de pausas passivas entre as séries. Diante disso, para aqueles que desejam executar os protocolos de ECIAI com maior segurança cardiovascular, sugerimos que seja realizado por indivíduos fisicamente ativos e que durante o exercício seja feito o controle da carga interna de treinamento por meio da PSE, $\mathrm{SpO}_{2}$ e FC. O programa de treinamento avaliado no presente estudo parece ser adequado para a melhora do condicionamento físico de praticantes avançados na prática de calistênia, ginástica, CrossFit $\circledast$, Cross Training, treinamento funcional e esportes em geral.

\section{Conclusão}

Diante do exposto, podemos concluir que o ECIAI foi capaz de provocar importantes ajustes cardiorrespiratórios agudos em adultos jovens com experiência nessa modalidade de exercício. Estes efeitos caracterizam um esforço físico vigoroso em indivíduos fisicamente ativos, reforçando a importância da utilização da PSE, $\mathrm{SpO}_{2}$ e FC para obter um melhor controle fisiológico da carga interna e uma maior segurança cardiovascular durante a sessão de treinamento. Com base na literatura e mediante as investigações do presente estudo, o nosso protocolo de treinamento também pode ser considerado como uma modalidade de alto esforço físico e que pode promover importantes demandas cardiovasculares mesmo em indivíduos com experiência avançada.

Sugere-se que estudos randomizados sejam realizados com o objetivo de avaliar os ajustes fisiológicos agudos e crônicos deste protocolo de exercício físico visando maior controle e segurança em sua aplicação prática, tanto para indivíduos sedentários quanto fisicamente ativos, como também, os seus riscos e benefícios para a saúde. 


\section{Agradecimentos}

Agradecemos pelo apoio financeiro do Programa de Pesquisa Produtividade do Centro Universitário Estácio de Sergipe. Em especial, agradecemos pelo apoio logístico na coleta de dados ao enfermeiro e técnico de laboratório Luís Carlos Ferreira São José.

\section{Referências}

Chaves, L. M. S. et al. (2020). Calisthenics and bodyweight exercises: different concepts or scientific synonyms? Rev Bras Fisiol Exerc. 2020 19(1):13-15. https://doi: https://doi.org/10.33233/rbfe.v19i1.3985.

Forjaz, C. L. et al. (1998). Post-exercise changes in blood pressure, heart rate and rate pressure product at different exercise intensities in normotensive humans. Braz J Med Biol Res. 1998. 31 (10): 1247-1255. https://doi: 10.1590/S0100-879X1998001000003.

Forjaz, C.L., \& Tinucci T. (2000). A medida da pressão arterial no exercício. Rev Bras Hipertens. 7(1): 79-87.

Foster, C. (1998). Monitoring training in athletes with reference to overtraining syndrome. Med Sci Sports Exerc. 30(7): 1164-8. https://doi: 10.1097/00005768-199807000-00023.

Galy, O. (2005). Is exercise-induced arterial hypoxemia in triathletes dependent on exercise modality? Int J Sports Med. 26 (9): 719-26. https://doi: 10.1055/s2005-837446.

Gist, N. H., Freese, E. C., \& Cureton, K. J (2014). Comparison of responses to two high-intensity intermittent exercise protocols. Journal of Strength and Conditioning Research. 28(11): 3033-40, https://doi: 10.1519/JSC.0000000000000522.

Kalra, S., \& Roitman, J. L. (2007). Exercise and acute cardiovascular events: placing the risks into perspective: a scientific statement from the american heart association council on nutrition, physical activity, and metabolism and the council on clinical cardiology. Journal of Cardiopulmonary Rehabilitation and Prevention. 27(4): 255-6. https://doi: 10.1097/01.HCR.0000281778.10468.d8.

Machado, A. F. et al. (2017). High-intensity interval training using whole-body exercises: training recommendations and methodological overview. Clin Physiol Funct Imaging. https://doi: 10.1111/cpf.12433.

McRae, G. et al. (2012). Extremely low volume, whole-body aerobic-resistance training improves aerobic fitness and muscular endurance in females. Appl Physiol Nutr Metab. 37 (6): 1124-31, https://doi: 10.1139/h2012-093.

Neto, G. R. et al. (2016). Acute resistance exercise with blood flow restriction effects on heart rate, double product, oxygen saturation and perceived exertion. Clin Physiol Funct Imaging. 36(1): 53-9. https://doi: 10.1111/cpf.12193.

Nummela, A., Hämäläinen, I., \& Rusko, H. (2002). Effect of hyperoxia on metabolic responses and recovery in intermittent exercise. Scand J Med Sci Sports. 12(5): 309-15. https://doi: 10.1034/j.1600-0838.2002.10157.x.

Paula, C. C. et al. (2020). Treinamento intervalado de alta intensidade (HIIT) como alternativa viável para induzir a prevenção de doenças respiratórias: um ponto de vista da imunologia do exercício durante o surto de COVID-19. Research, Society and Development, 9 (10), e7069109186. https://doi.org/10.33448/rsd-v9i10.9186

Peeling, P., \& Andersson, R. (2011). Effect of hyperoxia during the rest periods of interval training on perceptual recovery and oxygen re-saturation time. $J$ Sports Sci. 29 (2): 147-50. https://doi: 10.1080/02640414.2010.526133.

Santos, A. L. S et al. (2018). Comportamento da frequência cardíaca e da saturação de oxigênio durante um combate simulado de jiu-jitsu em participantes acima de 30 anos de idade. Revi Bras de Prescri e Fisiol do Exerc. 12 (74): 333-338.

Schaun, G. Z. et al. (2018). Whole-body high-intensity interval training induce similar cardiorespiratory adaptations compared with traditional high-intensity interval training and moderate-intensity continuous training in healthy men. Journal of Strength and Conditioning Research. 32 (10): 2730-42, https://doi: $10.1519 / \mathrm{JSC} .0000000000002594$.

Schaun, G. Z., \& Vecchio, F. B. D. (2018). High-intensity interval exercises' acute impact on heart rate variability: comparison between whole-body and cycle ergometer protocols. Journal of Strength and Conditioning Research. 32(1): 223-229, https://doi: 10.1519/JSC.0000000000002180.

Scott, B. R et al. (2015). Physical performance during high-intensity resistance exercise in normoxic and hypoxic conditions. J Strength Cond Res. 29 (3): $807-$ 15. https://doi: 10.1519/JSC.0000000000000680.

Thomas, J. R. et al. (2012). Métodos de pesquisa em atividade física. (6a ed.), Artmed, 2012.

Tucker, W. J. et al. (2015). Physiological responses to high-intensity interval exercise differing in interval duration. Journal of Strength and Conditioning Research. 29(12): 3326-35, https://doi: 10.1519/JSC.0000000000001000.

Weston, M. et al. (2014). Effects of low-volume high-intensity interval training (HIT) on fitness in adults: A Meta-Analysis of Controlled and Non-Controlled Trials. Sports Med. 44:1005-1017:13, https://doi: 10.1007/s40279-014-0180-z. 